\title{
Self Organization and Coordination
}

\author{
SCOTT E. PAGE \\ Center for Study of Complex Systems, University of Michigan, Ann Arbor, MI 48106, U.S.A.; \\ e-mail: spage@umich.edu
}

\begin{abstract}
In this paper, I analyze the organization of tasks or activities by a collection of agents. I begin by formally defining organized collections and robustly organized collections of agents in the context of a simple model. Within this framework, I demonstrate that organized collections and robustly organized collections exist and equilibria need not be organized. I then test whether adaptive agents can self-organize in this environment. I find that simple behavioral rules can lead to nearly organized, fairly robust collections of agents.
\end{abstract}

Key words: self-organization, coordination, equilibrium

Tens of millions of people making billions of decisions every week about what to buy and what to sell and where to work and how much to save and how much to borrow and what orders to fill and what stocks to accumulate and where to move and what schools to go to and what jobs to take and where to build supermarkets and movie theaters and electric power stations, when to invest in buildings above ground and mine shafts underground and fleets of trucks and ships and aircraft - if you are in a mood to be amazed, it can amaze you that the system works at all.

Thomas Schelling

Micromotives and Macrobehavior (p. 21)

\section{Introduction}

While long thought to require planning, order can also arise spontaneously. Birds form flocks, water particles swirl in eddies, and pedestrians in crowded train stations form rivers of traffic. This phenomenon is often referred to as 'order from the bottom-up'. Recently, scientists have attempted to explain this emergence of self-organized behavior using agent based models. Given the importance (and prevalence) of order in the economy, explaining it geographically (Krugman, 1996) and temporally have grown as research topics within economics (Kirman, 1997; Arthur and Durlauf, 1997). 
To date, researchers have succeeded in explaining the emergence of order in specific environments. Mitchell, Hraber, and Crutchfield (1993) demonstrated that locally informed one dimensional cellular automata can evolve identical local rules that collectively perform global calculations. And, Arthur (1994) in popularizing and recasting Schelling's (1978) beach problem as 'The Bar Problem', found it to be less of a problem than Schelling thought. Arthur reveals how an elaborate behavioral dance of adaptive actors can create collective predictability.

In this paper, I construct a minimalist multi-agent model of temporal task selection to see whether a collection of agents can self-organize in time and space without a central planner. The model can be interpreted as a multiple bar problem, or as a shopping problem where every agent must visit each of several establishments, but I prefer to think of it as an abstraction of more general coordination problems that occur within an economy. At the most basic level, language and technology must be coordinated in order for an economy to function (Durlauf, 1996). Property rights must not only be defined but be accepted. Norms of behavior and, more generally, culture also must be agreed upon to avoid crises and chaos (Axelrod, 1997).

The models created to study these situations typically include only two or three possible equilibria (Young, 1998). Some of these models rely on optimizing agents, but most use evolutionary arguments, assuming that reproduction favors actions generating higher payoffs (Kandori, Mailath, and Rob, 1993). These models demonstrate that evolution need not favor Pareto-dominant outcomes. In fact, the introduction of noise tends to lead the system toward risk dominant actions (Young, 1998). Among the other questions that have been asked and answered are how much time is spent in the bad equilibria (Blume, 1993) and how the topology of interactions influences the time spent in each equilibrium (Ellison, 1993).

The type of coordination that I model here differs. In some of the environments I consider, agents choose from among billions of strategies, so they cannot necessarily ponder all options instantaneously. In an economy, agents choose from a similarly large set (Matsuyama, 1992). Consider the definition of an ArrowDebreu commodity. It has physical characteristics, a location, and a time stamp. Economic coordination must occur along all of these dimensions. For example, a rancher selling cattle prefers to show up at the auction house when other sellers have stayed home. In addition, the set of commodities itself may be the result of a massive coordination problem with multiple equilibria, a point also made by Matsuyama (1992). As Schelling observes, the economy demonstrates 'amazing' levels of coordination given the dimensionality of the space of strategies. Some of this can be explained by the central limit theorem. To the extent that individual decisions depend upon additive random effects, there should be normal distributions in the aggregates, but not the small variances, i.e., the organization, commented upon by Schelling.

The model that I construct lends itself to both mathematical and computational techniques. In the model, agents choose routes among a finite set of locations, or 
alternatively sequences for performing a set of activities. Agents want to minimize the total attendance at the locations they visit - to avoid crowds. Organization requires all locations to have identical attendance levels in each time period. Such collections of routes exist and can be constructed easily, but the existence of organized collections of routes does not imply that decentralized agents responding to local information will necessarily form them. If they do, then, in the language of complex systems, the agents will have self-organized. In computational experiments, I find that artificial agents relying on simple rules can often self-organize. I also derive mathematical results that support these computational findings.

In studying whether decentralized agents self-organize, I also analyze the types of collections of routes they form. The collections depend upon the agents' behavioral rules and the starting population, but for the most part agents tend to find complicated collections of routes. In many cases, no two agents take the same route. This tremendous diversity might appear to be costly but in fact, it has an unintended benefit. Diverse collections prove robust to dropping individual locations. Comparing robustness for self-organized collections to minimal organized collections - the type of collections a central planner might choose - the former are far more robust. This occurs even though the agents do not take robustness into account when choosing their routes; this finding echoes the work of Norman Johnson (1998) who uncovers a relationship between individual level diversity and system level robustness in a graph traversing model.

I have organized the remainder of this paper into three sections. In the next section, I describe the basic model and present some mathematical results. In the middle section, I analyze self-organization both mathematically and computationally. In the computational model, I construct two types of agents that I call SIS agents and BRO agents. The former type rely on gradient based search, while the latter type rely on reproduction of the fittest. In the final section, I comment upon the larger research agenda to which this paper belongs.

\section{The Basic Model}

There are $M$ agents who must visit each of $L$ locations in $L$ time periods. I assume that an agent can visit only one location each period, so an agent's route, $r$, is an ordering of the $L$ locations. Agents want to minimize the total attendance at the locations they visit. A route can be considered as an element of $P(L)$, the permutation group on $L$ objects. A collection of routes for each agent $r=\left\{r_{1}, r_{2}, \ldots, r_{M}\right\}$ is organized if the same number of agents are at each location in every period. With one hundred agents and five locations, an organized collection of routes would require exactly twenty agents at each location in every period. Twenty agents visit location $\mathrm{A}$ in period 1 , twenty agents visit location $\mathrm{E}$ in period 3 , and so on. Throughout the paper, I will let letters denote locations and numbers denote periods where possible. ${ }^{1}$ 
Table I. A cyclic MICOR.

\begin{tabular}{lllllllll}
\hline Agent & \multicolumn{2}{l}{ Route } & & & & & & \\
\hline 1 & A & B & C & D & E & F & G & H \\
2 & B & C & D & E & F & G & H & A \\
3 & C & D & E & F & G & H & A & B \\
4 & D & E & F & G & H & A & B & C \\
5 & E & F & G & H & A & B & C & D \\
6 & F & G & H & A & B & C & D & E \\
7 & G & H & A & B & C & D & E & F \\
7 & H & A & B & C & D & E & F & G \\
\hline
\end{tabular}

Table II. A 'paired' MICOR.

\begin{tabular}{lllll}
\hline Agent & \multicolumn{2}{l}{ Route } \\
\hline 1 & AB & CD & EF & GH \\
2 & BA & DC & FE & HG \\
3 & CD & AB & GH & EF \\
4 & DC & BA & HG & FE \\
5 & EF & GH & AB & CD \\
6 & FE & HG & BA & DC \\
7 & GH & EF & CD & AB \\
8 & HG & FE & DC & BA \\
\hline
\end{tabular}

\subsection{ORGANIZED COLLECTIONS}

Constructing organized collections of routes is not difficult. The simplest require only $L$ different routes among the $M$ agents. I call these minimal collections of organized routes (MICORs). There are many types of MICORs as I now show for the case $L=8$. The most obvious MICORs are cyclic. Imagine placing the eight locations in a circle and assigning one agent to each location. In each time period move each agent one location in a clockwise direction. This creates a MICOR as shown in Table I.

But, MICORs can also be created by forming pairs of locations and implementing inter- and intra-pair switches as shown in Table II.

These two examples misleadingly suggest that MICORS must have a simple underlying organization. But, in fact, a MICOR can be a mangle of distinct routes as shown in Table III.

This third collection is harder to describe than the first two. So, as measured by minimal description length, the third collection is more complex. 
Table III. A baroque MICOR.

\begin{tabular}{lllllllll}
\hline Agent & \multicolumn{1}{l}{ Route } \\
\hline 1 & A & B & C & D & E & F & G & H \\
2 & B & C & A & E & F & D & H & G \\
3 & C & A & B & F & G & H & D & E \\
4 & G & H & D & A & B & C & E & F \\
5 & E & G & H & B & C & A & F & D \\
6 & H & F & E & G & D & B & C & A \\
7 & D & E & F & C & H & G & A & B \\
8 & F & D & G & H & A & E & B & C \\
\hline
\end{tabular}

Table IV.

\begin{tabular}{lllll}
\hline Agent & \multicolumn{2}{l}{ Route } \\
\hline 1 & A & B & C & D \\
2 & B & D & A & C \\
3 & B & C & D & A \\
4 & C & D & B & A \\
5 & C & A & D & B \\
6 & D & C & A & B \\
7 & D & A & B & C \\
8 & A & B & C & D \\
\hline
\end{tabular}

MICORs can be replicated or combined to form larger organized collections of routes, but MICORs cannot be thought of as the building blocks of organized collections of routes. As Example 1 shows, it is possible to construct an organized collection or routes that cannot be decomposed into MICORs. So, while some organized collections will contain MICORs or be multiple copies of a single MICOR, in general, MICORS are not a basis for organization.

EXAMPLE 1. The organized collection of routes as shown in Table IV cannot be decomposed into a disjoint set of MICORs.

In Table IV, there are eight routes and four locations, so exactly two agents will be at each location in every period. These eight routes cannot be broken into two sub collections of four routes in such a way that both groups will have exactly one agent at each location in each period. If it were possible to do so, one group would contain agent 1 , and the other would contain agent 8 , since the two agents take the same route. One sub collection must contain agent 3 with route BCDA. This group 
must also contain one of the agents (4 or 5) whose routes begin with location $C$. But, both of these agents go to the same location at the same time as agent 3, so the sub collection cannot be organized.

\subsection{DIVERSITY}

For a collection of routes to be organized, it must contain different routes. The least organized collections or routes contain agents all choosing the same route. Throughout this paper, I use a rather crude measure of diversity: the number of distinct routes selected by agents. This measure does not take into account the number of agents choosing each route. Consider the following collections of routes.

Collection 1: 97 agents take route $A B C D$, one takes route $B A D C$, one takes $C D A B$, and one takes DCBA.

Collection 2: 25 agents take route $A B C D, 25$ take route $B A D C, 25$ take $C D A B$, and 25 take $D C B A$.

Notice that the second collection has an even distribution across the four routes, while the first has only one agent taking each of three routes. Counting the number of distinct routes will not capture this spread. However, knowing whether the collection of routes is organized gives a signal as to the evenness of the spread. The second collection is organized whereas the first is not.

\subsection{ROBUSTNESS}

In addition to being organized, a collection of routes may be robust or it may be 'brittle' to dropping locations and time periods. By the latter, I mean that a collection may become highly disorganized after a location and time period are dropped. Intuitively, the robustness of a collection or routes should be related to the level of diversity. More diverse collections should be more robust. Some examples help to clarify this intuition. Consider the following three tables with organized collections of twenty four agents vising four locations. The first collection consists of six copies of each element of the permutation group on four objects.

Table V. Collection 1.

\begin{tabular}{ccccccc}
\hline Agents & Routes & & & & & \\
\hline $1-6$ & ABCD & ABDC & ACBD & ACDB & ADBC & ADCB \\
$7-12$ & BACD & BADC & BCAD & BCDA & BDAC & BDCA \\
$13-18$ & CABD & CADB & CBAD & CBDA & CDAB & CDBA \\
$21-24$ & DABC & DACB & DBAC & DBCA & DCAB & DCBA \\
\hline
\end{tabular}


The second collection consists of three copies of Example 1.

Table VI. Collection 2.

\begin{tabular}{ccccccccc}
\hline Agents & Routes & & & & & & & \\
\hline $1-8$ & ABCD & BDCA & BCDA & CDBA & CADB & DCAB & DABC & ABCD \\
$9-16$ & ABCD & BDCA & BCDA & CDBA & CADB & DCAB & DABC & ABCD \\
$17-24$ & ABCD & BDCA & BCDA & CDBA & CADB & DCAB & DABC & ABCD \\
\hline
\end{tabular}

The third collection consists of six copies of a simple cycle on four objects.

Table VII. Collection 3.

\begin{tabular}{ccccc}
\hline Agents & Routes & & & \\
\hline $1-4$ & ABCD & BCDA & CDAB & DABC \\
$5-8$ & ABCD & BCDA & CDAB & DABC \\
$9-12$ & ABCD & BCDA & CDAB & DABC \\
$13-16$ & ABCD & BCDA & CDAB & DABC \\
$17-20$ & ABCD & BCDA & CDAB & DABC \\
$21-24$ & ABCD & BCDA & CDAB & DABC \\
\hline
\end{tabular}

Now, suppose that location D closes and that the number of time periods reduces by one as well. Assume that the order in which an agent visits the remaining locations is the same as when the location D was included. For example, if the original route was ABCDEFG and location D is dropped, the route becomes ABCEFG. Given that convention, the collections can be rewritten as follows:

Table VIII. Collection 1.

\begin{tabular}{cllllll}
\hline Agents & \multicolumn{2}{l}{ Routes } & & & & \\
\hline $1-6$ & ABC & ABC & ACB & ACB & ABC & ACB \\
$7-12$ & BAC & BAC & BCA & BCA & BAC & BCA \\
$13-18$ & CAB & CAB & CBA & CBA & CAB & CBA \\
$21-24$ & ABC & ACB & BAC & BCA & CAB & CBA \\
\hline
\end{tabular}


Table IX. Collection 2.

\begin{tabular}{cllllllll}
\hline Agents & \multicolumn{2}{l}{ Routes } & & & & & & \\
\hline $1-8$ & ABC & BCA & BCA & CBA & CAB & CAB & ABC & ABC \\
$9-16$ & ABC & BCA & BCA & CBA & CAB & CAB & ABC & ABC \\
$17-24$ & ABC & BCA & BCA & CBA & CAB & CAB & ABC & ABC \\
\hline
\end{tabular}

Table X. Collection 3.

\begin{tabular}{cllll}
\hline Agents & \multicolumn{2}{l}{ Routes } & & \\
\hline $1-4$ & ABC & BCA & CAB & ABC \\
$5-8$ & ABC & BCA & CAB & ABC \\
$9-12$ & ABC & BCA & CAB & ABC \\
$13-16$ & ABC & BCA & CAB & ABC \\
$17-20$ & ABC & BCA & CAB & ABC \\
$21-24$ & ABC & BCA & CAB & ABC \\
\hline
\end{tabular}

Given these new routes, the attendance at each location in each time period becomes:

Table XI. Attendance.

\begin{tabular}{|c|c|c|c|c|c|c|c|c|c|}
\hline \multirow{2}{*}{$\begin{array}{l}\text { Collection } \\
\text { Number }\end{array}$} & \multicolumn{3}{|c|}{ Period 1} & \multicolumn{3}{|c|}{ Period 2} & \multicolumn{3}{|c|}{ Period 3} \\
\hline & $\mathrm{A}$ & $\mathrm{B}$ & $\mathrm{C}$ & $\mathrm{A}$ & $\mathrm{B}$ & $\mathrm{C}$ & $\mathrm{A}$ & $\mathrm{B}$ & $\mathrm{C}$ \\
\hline 1 & 8 & 8 & 8 & 8 & 8 & 8 & 8 & 8 & 8 \\
\hline 2 & 9 & 6 & 9 & 6 & 12 & 6 & 9 & 6 & 9 \\
\hline 3 & 12 & 6 & 6 & 6 & 12 & 6 & 6 & 6 & 12 \\
\hline
\end{tabular}

Notice the relationship between robustness and diversity. Collection 1, the most diverse collection, remains organized even after location $\mathrm{D}$ has been dropped, but the other two collections do not. Further, the second collection, which is more diverse than the third, has less variance in attendance after location $\mathrm{D}$ has been dropped. I define an organized collection of routes to be robustly organized with respect to location $k$ if when location $k$ and one time period are dropped, the collection remains organized. The next proposition states that robustly organized collections of routes exist. 
PROPOSITION 1. For any number of locations, there exist robustly organized collections of routes with respect to every location.

Proof. Consider the collection of routes that equals all possible permutations on $L$ objects. This collection contains $L$ ! routes. It suffices to show that this collection is robustly organized with respect to all $\ell \in\{1,2, \ldots L\}$ The proof to follow shows that dropping any location, creates $L$ copies of the permutation group on $L-1$ members, an organized collection of routes.

Without loss of generality assume that location $L$ is dropped. Consider all objects of the permutation group on $L$ objects that visit location $L$ first in their routes. There are $(L-1)$ ! such routes. After dropping location $L$, these routes comprise the permutation group on $(L-1)$ objects. This same holds for all other placements in the route: there are $(L-1)$ ! routes that visit $L$ second. After $L$ is dropped these form the permutation group on $(L-1)$ objects. It follows that after dropping location $L$, the collection of routes consists of $L$ copies of the permutation group on $(L-1)$ objects, an organized collection.

A collection can be robustly organized with respect to a specific location but not be equivalent to the permutation group or to some finite number of copies of the permutation group as the next example demonstrates.

EXAMPLE 2. Consider the following twelve routes. DABC, DCAB, DBCA, $A D B C, C D A B, B D C A, A B D C, C A D B, B C D A, A B C D, C A B D$, and BCAD. The attendance at each location in each time period equals three, so this collection of routes is organized. Now, drop location D. This leaves four copies of the following three routes: $A B C, C A B$, and $B C A$, which form a MICOR.

In the example, the location being dropped must be location $\mathrm{D}$. If location $\mathrm{C}$ were dropped the collection no longer remains organized. Define a collection that is robustly organized for any location as fully robust. The next proposition states that there exist fully robust collections of routes that are not copies of the permutation group.

PROPOSITION 2. There exist collections of $L$ ! routes that are robustly organized with respect to every location which are not the permutation group.

Proof. The proof is by construction of an example for the case $L=4$. Similar examples can be constructed for larger $L$. Begin with the $L !=24$ routes that form the permutation group on four objects.

Table XII.

\begin{tabular}{llllll}
\hline ABCD & ABDC & ACBD & ACDB & ADBC & ADCB \\
BACD & BADC & BCAD & BCDA & BDAC & BDCA \\
CABD & CADB & CBAD & CBDA & CDAB & CDBA \\
DABC & DACB & DBAC & DBCA & DCAB & DCBA \\
\hline
\end{tabular}


Consider the two pairs of routes (ABCD,ABDC) (BADC,BACD). In each pair, the second route can be created from the first (and vice versa) by flipping its third and fourth locations. To create a robustly organized collection of routes different from the permutation group, begin with the permutation group and replace the second route in each of the pairs with the first route.

It suffices to show that this change in the collection of routes remains organized. This requires showing that the routes are organized and remain so after dropping any of the four locations.

Organized: It suffices to show that the locations visited in each period for the sub collection of routes $\mathrm{ABCD}$ and $\mathrm{BADC}$ is the same as for for the subcollections of routes they replace, $\mathrm{ABDC}$ and $\mathrm{BACD}$. This follows by inspection.

Dropping location A: If location A is dropped, the new routes become: $\mathrm{BCD}$ and $\mathrm{BDC}$. The replaced routes become BDC and BCD.

Dropping location B: If location B is dropped, the new routes become: ACD and ADC. The replaced routes become ADC and ACD.

Dropping Location $C$ : If location $\mathrm{C}$ is dropped, the new routes become: $\mathrm{ABD}$ and $\mathrm{BAD}$. The replaced routes become ABD and BAD.

Dropping Location D: If location $\mathrm{D}$ is dropped, the new routes become: $\mathrm{ABC}$ and $\mathrm{BAC}$. The replaced routes become $\mathrm{ABC}$ and $\mathrm{BAC}$.

\subsection{SYSTEM LEVEL PHENOMENA AND INDIVIDUAL LEVEL CHOICES}

I have shown that the permutation group on $L$ objects is maximally diverse and also fully robust. And, I have shown that MICORs are not robustly organized. These two facts reveal a relationship between diversity and robustness. If a central planner were to coordinate routes without regard to robustness, she would probably construct a MICOR. If robustness were a consideration, she would construct the permutation group. The obvious question to ask next is what sort of collections will decentralized collections of agents evolve. Will they self-organize into diverse, robust collections or will they create fragile MICORs? The answer, as I shall show, depends upon how the collection of agents self-organizes upon how agents adopt their routes.

\section{Self-Organization}

I begin with a mathematical analysis of self-organization. First, I define an equilibrium with respect to route choice. I then prove that an equilibrium need not be 
self-organized. However, as I show later, self-organized collections will tend to be close to organized, given an appropriate distance metric.

Given a collection of routes, $r=\left\{r_{1}, r_{2}, \ldots r_{M}\right\}$, let the attendance for agent $i, A_{i}(r)$ equal the total attendance over all $L$ locations that agent $i$ visits, and let $A_{i \ell}(r)$ equal the attendance at the location that agent $i$ visits in the $\ell$ th period. Let $T_{\ell}^{s}(r)$ equal the attendance at location $\ell$ in period $s$.

Several issues arise in determining how agents choose routes. In this discussion, I will focus on just two: problem size and information. With twenty locations and twenty time periods there are $243,290,200,817,664,000$ possible routes. No agent could simultaneously contemplate all of them. However, if an agent knew the attendances at all twenty locations in each of the twenty time periods, a total of four hundred pieces of information, ${ }^{2}$ then she could formulate an integer programming problem and find the optimal route. ${ }^{3}$ Yet, both assumptions, knowing all of the location attendances and deriving the optimal route, seem unreasonable. Therefore, rather than assuming that agents best respond, I restrict the set of attainable routes, $h\left(r_{i}\right)$ to be a subset of the set of all possible routes.

Given this assumption, I can define a collection of routes $r=\left\{r_{1}, r_{2}, \ldots r_{M}\right\}$ to be an $h$-equilibrium if $A_{i}(r) \leq A_{i}\left(\hat{r}_{i}, r_{-i}\right)$ for all $\hat{r}_{i}$ in $h\left(r_{i}\right)$. In the case where $h\left(r_{i}\right)=P(L)$, this becomes a standard Nash Equilibrium. The next proposition states that any organized collection of routes is an $h$-equilibrium. When an organized collection of routes has been 'grown' by beginning from a non equilibrium state, I shall refer to the collection as self-organized.

PROPOSITION 3. Every organized collection of routes $\left\{r_{1}, r_{2}, \ldots r_{M}\right\}$ is an $h$-equilibrium for any $h$.

Proof. Suppose that agent $i$ changes its route from $r_{i}$ to $\hat{r}_{i}$. If the $\ell$ th location visited by agent $i$ is the same under both routes, $r_{i \ell}=\hat{r}_{i \ell}$, then attendances at that location are equal, $A_{i \ell}(r)=A_{i \ell}\left(\hat{r}_{i}, r-i\right)$. Otherwise, the attendance at the location on the route belonging to the self-organized collection must be less than on the other route, $A_{i \ell}(r)<A_{i \ell}\left(\hat{r}_{i}, r-i\right)$, which completes the proof.

The converse need not hold. A self-organized collection of routes need not be organized. The next example is a collection of routes that is an $h$-equilibrium for all $h$ but that is not organized.

EXAMPLE 3. Four agents, four locations:

Table XIII.

\begin{tabular}{ll}
\hline Agent & Route \\
\hline 1 & ABDC \\
2 & BCAD \\
3 & CDAB \\
4 & DBCA \\
\hline
\end{tabular}


This collection of routes, though not organized, is an $h$-equilibrium for all $h$. Each agent has an attendance of only one (itself) at three locations and is one of two agents at one other location during its route. Using the notation provided above, $A_{1}(r)=5, A_{2}(r)=5, A_{3}(r)=5$, and $A_{4}(r)=5$. To show that this collection of routes is an $h$-equilibrium for all $h$, it suffices to show that no agent has a route that reduces its attendance to 4 . To achieve this, agent 1 must visit location $\mathrm{A}$ in period 1 and in period 2, which violates the assumption that agents visit all locations. Agent 2 would have to visit location B in periods 1 and 3, also a violation of the assumptions. Similarly, agent 3 must visit location $B$ in periods 3 and 4, and agent 4 must visit location $A$ in both periods 2 and 4, completing the proof.

Though this example proves that equilibria need not be organized it does not tell how far from organized an equilibrium can be. The distance depends upon $h$, the heuristic for choosing new routes and upon the routes belonging to the collection. The next proposition states that if all routes belong to the collection, and if $h$ allows switches of pairs of locations, then any $h$-equilibrium is self-organized.

PROPOSITION 4. Suppose that $r$ is an $h$-equilibrium collection of routes and that $\left\{r_{i}\right\}_{i=1}^{M}=P(L)$, then the collection $r$ is self-organized, provided that $h$ allows switches of pairs of locations.

Proof. The proof is by contradiction. Without loss of generality assume that location $A$ has $k^{+}$agents at time period 1 , where $k^{+}>\frac{M}{L}$ and has $k^{-}$agents at time period 2, where $k^{-}<\frac{M}{L}$. An agent whose route begins AJ would NOT strictly prefer to switch to JA if its new attendance from the first two periods, $T_{A}^{2}(r)+$ $T_{J}^{1}(r)+2$ was weakly greater than his current attendance, $T_{A}^{1}(r)+T_{J}^{2}(r)$. Hereafter, to simplify notation, I drop the $(r)$ term. Since all routes belong to the collection this must hold for all $J$. In other words,

$$
\sum_{J \neq A}\left(T_{A}^{2}+T_{J}^{1}+2\right) \geq \sum_{J \neq A}\left(T_{A}^{1}+T_{J}^{2}\right)
$$

This can be rewritten as

$$
\left(\sum_{J \neq A} T_{J}^{1}-\sum_{J \neq A} T_{J}^{2}\right) \geq(L-1)\left(k^{+}-k^{-}-2\right) .
$$

Since $k^{+}>\frac{M}{L}$ and $k^{-}<\frac{M}{L}$, the right hand side is weakly positive. Also, because the total attendance in each period must sum to $M$,

$$
\sum_{J \neq A} T_{J}^{1}=\frac{M}{L}-k^{+}
$$


and

$$
\sum_{J \neq A} T_{J}^{2}=\frac{M}{L}-k^{-}
$$

It follows that the left hand side is strictly negative, a contradiction.

Note that this proposition does not say that if you begin with at least one copy of every possible route that you end up with a self-organized collection of agents. It only says that if you have an equilibrium that contains every possible route, then it is self-organized - provided that the route choosing heuristic allows pairs of locations to be exchanged. The next proposition and its corollary place bounds on the distance from self-organized an $h$-equilibrium can be when only pairs of locations can be switched.

PROPOSITION 5. Suppose that $r$ is an $h$-equilibrium collection of routes and that $h$ only allows switches of pairs of locations. Then, no agent whose total attendance exceeds $M$ can visit a location that has an attendance greater than $1+\left(\frac{M}{L}\right)$.

Proof. Assume that total attendance for agent $i, A_{i}(r)$ exceeds $M$. It suffices to show that the attendance at any location that agent $i$ visits cannot exceed $1+\frac{M}{L}$. Without loss of generality assume that the route $r_{i}$ visits the locations in increasing order. If no pair of locations can be switched to lower attendance, then the following inequalities must hold for $\ell=2, . . L$.

$$
T_{1}^{1}(r)+T_{\ell}^{\ell}(r) \leq T_{\ell}^{1}(r)+T_{1}^{\ell}(r)+2 .
$$

Summing these $(L-1)$ inequalities and dropping the $r$ gives the following inequality:

$$
\sum_{\ell=1}^{L} T_{\ell}^{\ell}+(L-2) T_{1}^{1} \leq \sum_{s=2}^{L} T_{1}^{s}+\sum_{\ell=2}^{L} T_{\ell}^{1}+(L-1) \cdot 2 .
$$

Since every agent visits each location, it follows that $\sum_{s=1}^{L} T_{1}^{s}=M$. And since in every period, each agent must be at some location $\sum_{\ell=1}^{L} T_{\ell}^{1}=M$. Further, by assumption $\sum_{\ell=1}^{L} T_{\ell}^{\ell}=A_{i}$, the attendance for the route of agent $i$. Therefore, the inequality above can be rewritten as

$$
A_{i}+(L-2) \cdot T_{1}^{1} \leq\left(M-T_{1}^{1}\right)+\left(M-T_{1}^{1}\right)+(L-1) \cdot 2
$$

which reduces to

$$
A_{i}+L \cdot T_{1}^{1} \leq 2(M+L-1) .
$$

By assumption, $A_{i}>M$, total attendance for the agent exceeds $M$. Therefore, this inequality can be rewritten

$$
L \cdot T_{1}^{1}<2(M+L-1)-M=M+2(L-1) .
$$


Dividing both sides by $L$ gives

$$
T_{1}^{1}<\frac{M}{L}+\frac{2(L-1)}{L} .
$$

Which implies $T_{1}^{1} \leq \frac{M}{L}+1$. Since this must hold for every location visited, the proof is complete.

This proposition can be used to place an upper bound on the total attendance for any agent.

COROLLARY 1. Suppose that $r$ is an $h$-equilibrium collection of routes and that $h$ only allows switches of pairs of locations. Then, no agent's total attendance can exceed $M+L-1$.

Proof. As before, without loss of generality assume that the agent visits the $L$ locations in ascending order. Suppose that each of the first $k$ locations on the route have an attendance of $\frac{M}{L}+1$. From the proof of the previous proposition recall that the following inequality holds for $\ell>1$.

$$
T_{1}^{1}(r)+T_{\ell}^{\ell}(r) \leq T_{\ell}^{1}(r)+T_{1}^{\ell}(r)+2 .
$$

Now suppose that $k=L$, and arrive at a contradiction. If $k=L$, then the inequalities can be rewritten as

$$
\frac{M}{L}+\frac{M}{L} \leq T_{\ell}^{1}(r)+T_{1}^{\ell}(r)
$$

As before, every agent must visit each location, so $\sum_{s=1}^{L} T_{1}^{s}=M$. And in every period, each agent must be at some location, so $\sum_{\ell=1}^{L} T_{\ell}^{1}=M$. So, summing these $L-1$ inequalities gives

$$
2(L-1) \cdot \frac{M}{L} \leq 2\left(M-\frac{M}{L}-1\right)
$$

which rearranged gives

$$
L \cdot \frac{M}{L} \leq(M-1) .
$$

This is a contradiction, therefore, $k<L$.

These mathematical results suggest that switching pairs should be an effective heuristic. 


\subsection{GROWING SELF-ORGANIZATION}

I now approach the question of self-organization computationally, trying to grow organized collections given specific rules for choosing routes. This mode of analysis emphasizes the gap between proving the existence of an equilibrium and constructing a world that achieves it. Phrased alternatively, taking a computational approach forces the modeller to have a complete representation. All assumptions about timing, information, or behavior must be included and implementable (Tesfatsion, 1997). Not only can a computational approach demonstrate the achievement of equilibrium, but when multiple equilibria exist, it can help to select from among them (Holland and Miller, 1988). Here, given the enormous number of equilibria, this will be valuable.

I attempt to grow self-organization using two types of agents. Under each, an agent $i$ switches from its initial route $r_{i}$ to some other route $\hat{r_{i}}$ if and only if $A_{i}(r)>$ $A_{i}\left(r_{-i} \hat{r}_{i}\right)$. The first type of agents rely on simple isolated switching (SIS). SIS agents switches pairs of locations in its route. The agent gets accurate information about whether attendance rose or fell and accepts the switch if the latter occurred.

It is a well known result that pairs of switches, such as those used by SIS agents, can be used to construct any route from any other route. However, this does not mean that if route $\hat{r}_{i}$ is preferred to $r_{i}$ that every switch from $r$ toward $\hat{r}_{i}$ lowers attendance. Using the notation from the previous section, given a route $r_{i}$, the set of neighboring routes $h\left(r_{i}\right)$ equals.

$$
h\left(r_{i}\right)=\left\{\hat{r}_{i}: \exists \ell, \ell^{\prime}, \text { s.t. } r_{i j}=\hat{r}_{i j} j \neq \ell, \ell^{\prime}, \hat{r}_{i \ell}=r_{i \ell^{\prime}} \text { and } \hat{r}_{i \ell^{\prime}}=r_{i \ell}\right\} .
$$

The second type of agent relies on a replicator dynamic. For convenience I call them best replicator operator agents, or BRO agents. In this case, $h\left(r_{i}\right)=$ $\left\{r_{j}\right\}_{j=1}^{M}$. An agent can pick any route from among those in the current collection. Theoretically, all routes are possible; however, in any particular experiment, the only available routes are those that exist in the population. The lack of mutation bounds the level of diversity to that present in the initial population.

\subsection{SELF-ORGANIZING WITH BRO AND SIS}

The natural statistic to gather is the probability that self-organization occurs from random starting points. The data suggest that SIS agents outperform BRO agents. I begin with 120 agents and vary the number of locations from 3 to 20, including only those numbers that divide evenly into 120 . (Otherwise, self-organization is not possible.) So, this means that $L$ belongs to the set $\{3,4,5,6,8,10,12,15,20\}$. In each time segment, I update $M$ randomly chosen agents (some agents may be picked more than once) sequentially. This guarantees that agents can take into account the changes in the routes made by other agents during that time segment. I run the experiments for one hundred time segments, so on average, each agent can change its route one hundred times. 
SIS agents demonstrate an 'amazing' (in the Schelling sense of the word) ability to self-organize. In Table XIV, I show the percentage of time that SIS agents selforganize and the number of time segments until equilibrium.

\subsubsection{Agents}

For the initial seeding, I began with a common route and allowed up to four hundred changes of pairs of locations. The number of location pairs switched was uniform in the set $\{0,1, \ldots 400\}$. In the tables that follow, all data are averages over one hundred trials. The second column gives the percentage that successfully self-organized and the third column gives the number of time segments (up to one hundred) until all collections were self-organized. This means that in each of the one hundred trials, SIS agents had self-organized in first three periods.

Table XIV. SIS self-organization.

\begin{tabular}{crr}
\hline \# Loc's & $\%$ S-O & \multicolumn{1}{c}{ TS } \\
\hline 3 & 100 & 3 \\
4 & 100 & 9 \\
5 & 100 & 12 \\
6 & 100 & 31 \\
8 & 80 & 100 \\
10 & 50 & 100 \\
12 & 8 & 100 \\
15 & 0 & 100 \\
20 & 0 & 100 \\
\hline
\end{tabular}

These data show that as the number of locations grows, SIS agents become less likely to self-organization. This is not too surprising. The number of non selforganized equilibria might increase in the number of locations, because there are more subsets of locations on which agents could get stuck in a bad equilibrium.

As Table XV shows, BRO agents self-organize less often.

This occurs because of a lack of diversity. A BRO agent may choose a suboptimal route because no other agent in the population is taking a better one. So the system gets stuck.

The percentage of self-organized collections is a rather crude measure. It could be that the BRO agents are locating nearly self-organized collections of routes. To test this hypothesis, I define the distance from organized for a collection, $r$, to be the average attendance across agents minus the average in an organized collection, $\frac{M}{L}$.

$$
d(r)=\frac{1}{L^{2}}\left(\sum_{\ell=1}^{L} \sum_{s=1}^{L}\left(T_{\ell}^{s}\right)^{2}\right)-\frac{M}{L} .
$$


Table XV. BRO self-organization.

\begin{tabular}{crr}
\hline \# Loc's & $\%$ S-O & \multicolumn{1}{c}{ TS } \\
\hline 3 & 100 & 1 \\
4 & 100 & 11 \\
5 & 80 & 100 \\
6 & 6 & 100 \\
8 & 0 & 100 \\
10 & 0 & 100 \\
12 & 0 & 100 \\
15 & 0 & 100 \\
20 & 0 & 100 \\
\hline
\end{tabular}

Table XVI. Distance from self-organization (100 iterations).

\begin{tabular}{clll}
\hline \# Loc's & SIS & BRO & RAN \\
\hline 3 & 0.000 & 0.000 & 0.613 \\
4 & 0.000 & 0.000 & 0.795 \\
5 & 0.000 & 0.001 & 0.793 \\
6 & 0.000 & 0.006 & 0.832 \\
8 & 0.001 & 0.035 & 0.846 \\
10 & 0.002 & 0.169 & 0.908 \\
12 & 0.004 & 0.335 & 0.913 \\
15 & 0.010 & 0.570 & 0.951 \\
20 & 0.021 & 0.784 & 1.045 \\
\hline
\end{tabular}

Table XVI presents the data on the average distance from organized for 120 SIS and BRO agents respectively for varying numbers of locations. It also shows the average distance from organized for randomly generated collections of routes. These data show that, SIS agents come close to self-organization even when there are a large number of locations. Further, BRO agents perform well when the number of locations is small and badly when the number of locations is high.

As the number of locations grows large, the BRO agents' performance drops off sharply. This is because the BRO agents can choose from among at most 120 of the many routes available, fewer than one in thirty thousand of the possible routes. The fact that BRO agents get close at all is remarkable.

Counting the number of distinct routes in the final collections shown in Table XVII reveals that SIS agents evolve much more diverse collections than 
Table XVII. Diversity \# distinct routes.

\begin{tabular}{ccl}
\hline \# Loc's & \%SIS & BRO \\
\hline 3 & $* 6$ & $* 6$ \\
4 & 23.94 & 23.72 \\
5 & 78.90 & 63.20 \\
6 & 112.52 & 75.68 \\
8 & 119.94 & 66.52 \\
10 & $* 120$ & 62.06 \\
12 & $* 120$ & 68.10 \\
15 & $* 120$ & 76.48 \\
20 & $* 120$ & 86.52 \\
\hline
\end{tabular}

do BRO agents. Those entries with asterisks $(*)$ signify the collections that are maximally diverse given the population size.

All of the BRO agents diversity comes from the initial collection of routes. A less diverse initial collection would hamper the BRO agents far more than it would the SIS agents. In fact, even if all routes initially were identical, the SIS agents can self-organize. In an experiment with four locations and 120 agents, in each of one hundred trials SIS agents had located a self-organized collection by time segment fourteen, only three more time segments than when the initial population was diverse.

\subsubsection{Agents}

Since a lack of diversity lowers the BRO agent's performance. An increase in the number of agents from 120 to 600 should benefit the BRO agents. The effects on SIS agents are not immediately obvious. On the one hand, more agents could take longer. On the other hand, the larger number could create better averaging and therefore less time to organization. As before, I allow for the number of agent updates in each time segment to equal the number of agents. I find that with more agents, self-organization occurs more quickly.

As expected, BRO agents perform far better with 600 agents than with 120 .

The data on distance to self-organization are even more compelling. In the case of 15 locations, the distance falls to 0.02 from 0.57 and with 20 locations, the distance falls to 0.14 from 0.784 . In each case, the initial distances from self-organization are approximately the same.

\subsection{SCALING}

As a further check on how the system scales, I ran computational experiments with up to six thousand agents and twenty locations. The data presented in the table 
Table XVIII. SIS self-organization.

\begin{tabular}{ccr}
\hline \# Loc's & $\%$ S-O & \multicolumn{1}{c}{ TS } \\
\hline 3 & 100 & 1 \\
4 & 100 & 2 \\
5 & 100 & 4 \\
6 & 100 & 10 \\
8 & 100 & 24 \\
10 & 100 & 54 \\
12 & 100 & 64 \\
15 & 88 & 100 \\
20 & 38 & 100 \\
\hline
\end{tabular}

Table XIX. BRO self-organization.

\begin{tabular}{crr}
\hline \# Loc's & $\%$ S-O & \multicolumn{1}{c}{ TS } \\
\hline 3 & 100 & 1 \\
4 & 100 & 2 \\
5 & 100 & 23 \\
6 & 92 & 100 \\
8 & 0 & 100 \\
10 & 0 & 100 \\
12 & 0 & 100 \\
15 & 0 & 100 \\
20 & 0 & 100 \\
\hline
\end{tabular}

below are for SIS agents. Given the increase in the number of agents, I increased the maximal number of switches of pairs in the creation of the random routes from four hundred to six hundred.

\subsection{THE SPEED OF SELF-ORGANIZATION}

These systems of agents self-organize quickly. In this section, I derive some mathematical results which explain the rate of convergence. To bound the rate of convergence, I reinterpret the distance function as an energy function. It can then be shown that when an agent changes its route, lowering its average attendance, it also reduces the total energy in the system. Hereafter, I shall refer to energy as disorganization. 
Table XX. SIS self-organization.

\begin{tabular}{rrr}
\hline \# M & $\%$ S-O & \multicolumn{1}{c}{ TS } \\
\hline 1000 & 3 & 100 \\
2000 & 28 & 100 \\
3000 & 98 & 100 \\
4000 & 99 & 100 \\
5000 & 100 & 44 \\
6000 & 100 & 39 \\
\hline
\end{tabular}

Formally, define the disorganization, $D(r)$ of a collection of routes to be the sum of the squares of the attendances at each time in each location. Note that disorganization differs from distance to organization by a constant: $D(r)=d(r)+$ $\frac{M}{L}$.

$$
D(r)=\sum_{\ell=1}^{L} \sum_{s=1}^{L}\left(T_{\ell}^{s}(r)\right)^{2} .
$$

The next proposition states that if an agent locates an improvement in its route, it lowers the level of disorganization.

PROPOSITION 6. Given a collection of routes $r$, if $A_{i}(r)>A\left(r_{i}^{\prime}, r_{-i}\right)$, and $r_{i}^{\prime}$ is created from $r_{i}$ by switching a single pair of locations, then $D(r)>D\left(r_{i}^{\prime}, r_{-i}\right)$.

Proof. Without loss of generality, suppose that locations A and B were originally visited in periods 1 and 2 respectively, but that now location $B$ is visited in period 1, and location A is visited in period 2. Since the new route has lower total attendance, it follows that

$$
T_{A}^{1}+T_{B}^{2}<T_{B}^{1}+T_{A}^{2}+2 .
$$

By definition $D(r)-D\left(r_{i}^{\prime}, r_{-1}\right)$ equals

$$
\begin{aligned}
& \left(T_{A}^{1}\right)^{2}+\left(T_{B}^{2}\right)^{2}+\left(T_{B}^{1}\right)^{2}+\left(T_{A}^{2}\right)^{2}- \\
& \quad-\left(T_{A}^{1}-1\right)^{2}-\left(T_{B}^{2}-1\right)^{2}-\left(T_{B}^{1}+1\right)^{2}-\left(T_{A}^{2}+1\right)^{2} .
\end{aligned}
$$

Which reduces to

$$
D(r)-D\left(r_{i}^{\prime}, r_{-1}\right)=2 T_{B}^{1}+2 T_{A}^{2}-2 T_{A}^{1}-2 T_{B}^{2}++4 .
$$

By assumption $T_{A}^{1}+T_{B}^{2}<T_{B}^{1}+T_{A}^{2}+2$. Therefore, the right hand side is positive, which implies that $D(r)$ decreases.

Building upon this proof, a crude upper bound can be constructed for the maximal number of improvements that can be made by switching pairs of locations. 
PROPOSITION 7. If h allows only switches of pairs of locations, then at most $\frac{\left(L^{2}-1\right) M^{2}}{2}$ route improvement can be made before attaining an equilibrium.

Proof. The maximum possible value for $D(r)$ occurs when all agents take the exact same route. In this case, $D(r)=L^{2} \cdot M^{2}$. The minimal value is attained when there are exactly $\frac{M}{L}$ agents at each location in each time period, which implies that $D(r)=M^{2}$. From the proof of the previous proposition, any switch of pairs of locations that lowers the agents total attendance, lowers $D(r)$ by at least 2, which completes the proof.

The proposition describes a worst case scenario at odds with the average finding from the computational experiments. The proposition states that as the population grows the time to convergence increases by order $M^{2}$. If $M$ agents update their routes each time segment, then this would mean that the number of time segments until convergence should increase linearly in the number of agents. But, in computational experiments, convergence decreases in $M$. A partial explanation for the variance between the worse case and what happens on average can be provided by an appeal to the central limit theorem. With lots of agents, the percentage of agents in each location will approach $\frac{M}{L}$ in each time period. And, since the number of agent updates in each time segment equals the total number of agents, the random starting points are likely to organize more quickly. In other words, with one hundred agents and twenty locations, the populations at each location in each period may vary from two to ten percent of the population, but with thousands of agents, the populations at each location in each time period will be close to five percent. Thus, the large populations will organize more quickly. ${ }^{4}$ The extension of this logic to the case of the millions and million of which Schelling speaks explains how the amazing occurs.

\subsection{ROBUSTNESS}

I next analyze the robustness of self-organized collections of routes. Recall that I measure robustness according to whether the system remains organized after a location and time period have been dropped. Robustness is a system level characteristic. An agent in an economy would probably not know the robustness of its strategy unless he noticed that he was seeing many of the same people at the different locations, which would suggest over-coordination.

To test the robustness of the collections, I allow agents to self-organize over six, five and four locations and then drop one location. I find that in none of the one hundred cases for each number of initial locations do the agents evolve a robust organization. This is not surprising given the rarity of robustly organized collections. The more relevant data are the distances from organization.

The SIS agents tend to be much closer to self-organized than a random collection of agents. Interestingly, in the aforementioned case with four sites and the initial population of 120 identical routes where the SIS agents self-organized in 
Table XXI. Robustness: Distance from self-organization after dropping a location.

\begin{tabular}{llll}
\hline \# Init Loc's & SIS & BRO & RAN \\
\hline 4 & 0.148 & 0.202 & 0.508 \\
5 & 0.170 & 0.307 & 0.602 \\
6 & 0.212 & 0.450 & 0.707 \\
\hline
\end{tabular}

Table XXII. Attendance.

\begin{tabular}{|c|c|c|c|c|c|c|c|c|}
\hline \multicolumn{3}{|c|}{ Period 1} & \multicolumn{3}{|c|}{ Period 2} & \multicolumn{3}{|c|}{ Period 3} \\
\hline $\mathrm{A}$ & B & $\mathrm{C}$ & A & $\mathrm{B}$ & $\mathrm{C}$ & $\bar{A}$ & $\mathrm{~B}$ & $\mathrm{C}$ \\
\hline 30 & 30 & 60 & 60 & 60 & 0 & 30 & 30 & 60 \\
\hline
\end{tabular}

fourteen or fewer periods, the level of robustness was not significantly different than with the diverse initial population. Looking at an example helps to explain SIS agents performed better according to this robustness measure. In a randomly chosen set of output, 31 of the BRO agents had chosen the route ABDC, 30 had chosen the route BACD, 29 had chosen CDAB, and 30 had chosen DCBA. Notice that ABDC, BACD, CDAB, and DCBA forms a MICOR. What happens when you drop location $\mathrm{D}$ ? You obtain the following four routes $\mathrm{ABC}, \mathrm{BAC}, \mathrm{CAB}$, and CBA. To simplify the mathematics, assume that there are thirty agents taking each route. The attendances are given in Table XXII.

In this example, the distance to organization equals 3.333. This is less organized than a random collection of routes, which in the table above is shown to have a distance of only 0.508 .

\section{Conclusion}

In this paper, I have constructed a model to study self-organization. I have demonstrated how unsophisticated agents can self-organize or come close to doing so. And, have found that self-organization becomes easier as the number of agents increases and harder when the number of locations increases. I showed that the collections of routes that agents evolve are diverse, and that this diversity makes them robust to dropping locations. Finally, I have supported these computational findings with mathematical results.

The particulars of the model, agents choosing routes to visit locations, though rather mundane, fit within a broader research enterprise: the study of decentralized coordination. Investigations of how socio-economic systems self-organize, 
whether they be national economies or corner market places are relatively rare. Yet, the level and extent of organization in the economy can be awe inspiring. The organized flow of goods to markets and people in a crowded city, the elaborate dance of waiters, waitresses, and busboys in a busy restaurant, and the stream of cars along cities streets occur with only partial orchestration. This paper belongs to a research agenda that hopes to strip away the mysteries of that self-organization; to explain how decentralized systems of heterogeneous agents can arrange themselves in efficient ensembles. In the past, these questions have been ignored because scholars had few ways to analyze systems with large numbers of diverse agents. Agent based computational models allow the construction of order from the bottom up; a researcher can generate artificial worlds which exhibit both order and disorder. The resulting research question of whether self-organization will prove successful, critical (Bak 1996), or catastrophic may even someday provide as much grist for the intellectual mill as has the question of whether unfettered markets will produce efficient outcomes.

\section{Acknowledgements}

The author would like to thank Ken Kollman and Norman Johnson for their input on this and related projects.

\section{Notes}

${ }^{1}$ For some general proofs, I rely on lower case letters to denote both locations and periods. I apologize for any inconvenience this causes to the reader.

2 Actually, 361 pieces, because once the attendances at nineteen locations are known in each of the first nineteen periods, the twentieth can be deduced. Further, once the first nineteen period attendances are known, the twentieth period attendances can also be deduced, since each location must have the same total attendance.

${ }^{3}$ The encoding of the problem is obvious. Let $x_{i j}=1$ if the agent visits location $j$ in period $i$ and write the appropriate constraints and objective function.

${ }^{4}$ Of course, many of the populations will not self-organize fully, but they will come close.

\section{References}

Arthur, W.B. (1994). The bar problem. American Economic Review: Papers and Proceedings, 84.

Arthur, B., Durlauf, S. and Lane, D. (1997). The Economy as an Evolving Complex System II. Addison Wesley, Redwood City, CA.

Axelrod, R. (1997). The Complexity of Cooperation. Princeton University Press, Princeton.

Bak, P. (1996). How Nature Works: The Science of Self-Organized Criticality. Springer Verlag, New York.

Blume, L. (1993). The statistical mechanics of strategic interaction. Games and Economic Behavior, 5, 387-426.

Durlauf, S. (1996). Statistical mechanics approaches to socioeconomic behavior. Santa Fe Institute Working Paper 96-08-069.

Ellison, G. (1993). Learning, local interaction, and coordination. Econometrica, 61, 1047-1072. 
Holland, J. and Miller, J. (1991). Artificial agents in economic theory. American Economic Review Papers and Proceedings, 81, 365-370.

Johnson, N.L. (1998). Collective problem solving: Functionality beyond the individual. Los Alamos Working Paper LA-UR-98-2227.

Kalai, E. and Lehrer, E. (1993). Rational learning leads to nash equilibrium. Econometrica, 61, 11091045.

Kandori, M., Mailath, G. and Rob, R. (1993). Learning, mutation, and long run equilibria in games. Econometrica, 61, 29-56.

Kirman, A. (1997). The economy as an interactive system. In W.B. Arthur, S. Durlauf and D. Lane (eds.), The Economy as a Complex Evolving System II, 491-533. Addison Wesley, Reading, MA.

Krugman, P. (1996). The Self-Organizing Economy. Blackwell, Cambridge.

Matsuyama, K. (1992). Economic development as coordination problems. Mimeo Northwestern University.

Mitchell, M., Hraber, P.T. and Crutchfiel, J.P. (1993). Revisiting the edge of chaos: Evolving cellular automata to perform computations. Complex Systems, 7, 89-130.

Schelling, T. (1978). Micromotives and Macrobehaviour. W.W. Norton, New York.

Tesfatsion, L. (1997). How economists can get a-life. In W.B. Arthur, S. Durlauf and D. Lane (eds.), The Economy as a Complex Evolving System II, 533-565. Addison Wesley, Reading, MA.

Young, H.P. (1998). Individual Strategy and Social Structure: An Evolutionary Theory of Institutions. Princeton University Press. 